\title{
LENGTH COMPLEXITY OF TENSOR PRODUCTS
}

\author{
WOLMER V. VASCONCELOS
}

\begin{abstract}
In this paper we introduce techniques to gauge the torsion of the tensor product $A \otimes_{R} B$ of two finitely generated modules over a Noetherian ring $R$. The outlook is very different from the study of the rigidity of Tor carried out in the work of Auslander ([1]) and other authors. Here the emphasis in on the search for bounds for the torsion part of $A \otimes_{R} B$ in terms of global invariants of $A$ and of $B$ in special classes of modules: vector bundles and modules of dimension at most three.
\end{abstract}

\section{CONTEnts}

1. Introduction

2. Preliminaries

3. Dimension 1

4. Vector bundles

5. Dimension 2

6. Dimension 3

7. Graded modules

8. Some open questions

References

\section{INTRODUCTION}

Let $R$ be an integral domain and $A$ and $B$ finitely generated, torsionfree $R$-modules. It is a challenging task to determine whether the tensor product $A \otimes_{R} B$ is also torsionfree, and if not, what are the invariants of its torsion submodule. It was a remarkable discovery by M. Auslander ([1]; see also [8]) that for regular local rings the torsionfreeness of $A \otimes_{R} B$ makes great but precise demands on the whole homology of $A$ and of $B$. These results have been taken up by other authors, with an important development being [7] with its extensions to various types of complete intersections.

Date: October 27, 2018.

Partially supported by the NSF.

Key words and phrases: Castelnuovo-Mumford regularity, cohomological degree, graded module, torsion module, vector bundle.

2000 Mathematics Subject Classification: 13H10, 13H15, 13D07, 13D30. 
The focus of the study of the torsion of $A \otimes_{R} B$ carried out here is very different from that of the rigidity of Tor carried out in the works mentioned above. The overall goal is that of determining bounds for the torsion part of $A \otimes_{R} B$ in terms of invariants of $A$ and of $B$. Let $(R, \mathfrak{m})$ be a Noetherian local ring, and let $A$ and $B$ be finitely generated $R$-modules. The HomAB question, first treated in [4] and further developed in [5], asks for estimates for the number of generators of $\operatorname{Hom}_{R}(A, B)$ in terms of invariants of $A$ and $B$ (or even of $R$ ). Since the answer in special cases depends on cohomological properties of $A$ and $B$, it seems appropriate to express bounds for $\nu\left(\operatorname{Hom}_{R}(A, B)\right)$ in terms of some extended multiplicity functions $\operatorname{Deg}(A)$ and $\operatorname{Deg}(B)$ of $A$ and $B$ (see [6]).

Here we consider an analogue of the HomAB question for tensor products which we formulate as follows:

Question 1.1. Can the torsion of a tensor product be estimated in terms of multiplicity invariants of $A$ and $B$ ? In particular, how to approach the calculation of $\lambda\left(H_{\mathfrak{m}}^{0}\left(A \otimes_{R} B\right)\right)$ or the related $\nu\left(H_{\mathfrak{m}}^{0}\left(A \otimes_{R} B\right)\right)$ ? In this note we look for the existence of polynomials $\mathbf{f}(x, y)$ with rational coefficients depending on invariants of $R$ such that

$$
h_{0}\left(A \otimes_{R} B\right)=\lambda\left(H_{\mathfrak{m}}^{0}\left(A \otimes_{R} B\right)\right) \leq \mathbf{f}(\operatorname{Deg}(A), \operatorname{Deg}(B)) .
$$

More generally, we look for similar bounds for

$$
h_{0}\left(H_{\mathfrak{m}}^{0}\left(\operatorname{Tor}_{i}^{R}(A, B)\right)\right), i \geq 0 .
$$

We shall consider special cases of these questions but in dimensions $\leq 3$, vector bundles and some classes of graded modules. The most sought after kind of answer for the shape of $h_{0}(A \otimes B)$ has the format

$$
h_{0}(A \otimes B) \leq c(R) \cdot \operatorname{Deg}(A) \cdot \operatorname{Deg}(B),
$$

where $c(R)$ is a function depending on the dimension of $R$ and some of its related invariants such as its Betti numbers. Two of such results are: (i) first Theorem 4.2 that asserts that $c(R)=\operatorname{dim} R$ works if $R$ is a regular local ring and $A$ is a module free on the punctured spectrum, then (ii) Theorem 6.1 that establishes a similar result, with $c(R)<4$, if both $A$ and $B$ are torsionfree modules over a 3-dimensional regular local ring.

The difficulties mount rapidly if both $A$ and $B$ have torsion. Thus in Theorem 7.2 , if $A$ is a graded torsion module over $k[x, y]$, generated by elements of degree zero, then the best we have achieved is to get $h_{0}(A \otimes A)<\operatorname{hdeg}(A)^{6}$.

On the positive side, one general argument (Theorem 2.7) will show that any polynomial bound for $h_{0}(A \otimes B)$ leads to a similar bound for $h_{0}\left(\operatorname{Tor}_{i}^{R}(A, B)\right)$, when the cohomological degree function $\operatorname{hdeg}(\cdot)$ is used. 


\section{Preliminaries}

Throughout $(R, \mathfrak{m})$ is either a Noetherian local ring or a polynomial ring over a field. For unexplained terminology we refer to [2]. For simplicity of notation, we often denote the tensor product of the $R$-modules $A$ and $B$ by $A \otimes B$. For a module $A, \nu(A)$ will denote its minimal number of generators. If $A$ is a module with a composition series, we denote its length by $\lambda(A)$. Two sources, where some of the techniques used with cohomological degrees were applied to the HomAB questions, are [4] and [5].

Finite support. We will use the following notation. Set $H_{\mathfrak{m}}^{0}(E)=E_{0}$, and $h_{0}(E)=$ $\lambda\left(E_{0}\right)$. More generally, if $H_{\mathfrak{m}}^{i}(E)$ has finite length, we set $h_{i}(E)=\lambda\left(H_{\mathfrak{m}}^{i}(E)\right)$.

Let us begin with the following two observations.

Proposition 2.1. Let $(R, \mathfrak{m})$ be a Noetherian local ring and $A, B$ finitely generated $R$-modules. Then

$$
\begin{aligned}
h_{0}\left(A \otimes_{R} B\right) & \leq h_{0}(A) \cdot \nu(B)+h_{0}(B) \cdot \nu(A)+h_{0}\left(A / A_{0} \otimes_{R} B / B_{0}\right), \\
\nu\left(H_{\mathfrak{m}}^{0}\left(A \otimes_{R} B\right)\right) & \leq \nu\left(A_{0}\right) \cdot \nu(B)+\nu(A) \cdot \nu\left(B_{0}\right)+\nu\left(H_{\mathfrak{m}}^{0}\left(A / A_{0} \otimes_{R} B / B_{0}\right) .\right.
\end{aligned}
$$

The other reduction involves replacing $A$ and $B$ by their direct sum $A \oplus B$. This is allowed because the invariants of $A$ and $B$ are essentially additive, and the analysis of $A \otimes A$ is often simpler than that of $A \otimes B$.

Big Degs. Clearly, to estimate $h_{0}(A \otimes B)$, the knowledge $h_{0}(A)$ and $h_{0}(B)$ are far from enough. Even more strongly, data provided from the multiplicities $\operatorname{deg}(A)$ and $\operatorname{deg}(B)$ will also fall far short of the goal. We will focus instead on the so-called extended or cohomological degree functions of [6]. (See [12, Section 2.4] for a discussion.)

A part to the definition of these functions is a method to select appropriate hyperplane sections, a process that may require that the residue fields of the rings be infinite. The notions apply to standard graded algebras or local rings, as the following definitions illustrate.

Definition 2.2. A cohomological degree, or extended multiplicity function, is a mapping from the category $\mathcal{M}(R)$ of finitely generated $R$-modules,

$$
\operatorname{Deg}(\cdot): \mathcal{M}(R) \mapsto \mathbb{N}
$$

that satisfies the following conditions.

(i) If $L=H_{\mathfrak{m}}^{0}(M)$ is the submodule of elements of $M$ that are annihilated by a power of the maximal ideal and $\bar{M}=M / L$, then

$$
\operatorname{Deg}(A)=\operatorname{Deg}(\bar{M})+\lambda(L)
$$

where $\lambda(\cdot)$ is the ordinary length function. 
(ii) (Bertini's rule) If $M$ has positive depth, there is $h \in \mathfrak{m} \backslash \mathfrak{m}^{2}$, such that

$$
\operatorname{Deg}(M) \geq \operatorname{Deg}(M / h M)
$$

(iii) (The calibration rule) If $M$ is a Cohen-Macaulay module, then

$$
\operatorname{Deg}(M)=\operatorname{deg}(M)
$$

where $\operatorname{deg}(M)$ is the ordinary multiplicity of $M$.

These functions will be referred to as big Degs. If $\operatorname{dim} R=0, \lambda(\cdot)$ is the unique Deg function. For $\operatorname{dim} R=1, \operatorname{Deg}(A)=\lambda(L)+\operatorname{deg}(A / L)$. When $d \geq 2$, there are several big Degs. An explicit Deg, for all dimensions, was introduced in [11]. It has a recursive aspect.

Definition 2.3. Let $M$ be a finitely generated graded module over the graded algebra $A$ and $S$ a Gorenstein graded algebra mapping onto $A$, with maximal graded ideal $\mathfrak{m}$. Set $\operatorname{dim} S=r, \operatorname{dim} M=d$. The homological degree of $M$ is the integer

$$
\begin{aligned}
\operatorname{hdeg}(M)= & \operatorname{deg}(M)+ \\
& \sum_{i=r-d+1}^{r}\left(\begin{array}{c}
d-1 \\
i-r+d-1
\end{array}\right) \cdot \operatorname{hdeg}\left(\operatorname{Ext}_{S}^{i}(M, S)\right) .
\end{aligned}
$$

This expression becomes more compact when $\operatorname{dim} M=\operatorname{dim} S=d>0$ :

$$
\begin{aligned}
\operatorname{hdeg}(M)= & \operatorname{deg}(M)+ \\
& \sum_{i=1}^{d}\left(\begin{array}{l}
d-1 \\
i-1
\end{array}\right) \cdot \operatorname{hdeg}\left(\operatorname{Ext}_{S}^{i}(M, S)\right) .
\end{aligned}
$$

We are going to recall some of the bounds afforded by a Deg function.

Theorem 2.4 ([12, Theorem 2.94]). For any Deg function and any finitely generated $R$-module $M$,

$$
\beta_{i}(M) \leq \operatorname{Deg}(M) \cdot \beta_{i}(k),
$$

where $\beta_{i}(\cdot)$ is the ith Betti number function.

Theorem 2.5 ([9]). Let $A$ be an standard graded algebra over an infinite field and let $M$ be a nonzero finitely generated graded $A$-module. Then for any $\operatorname{Deg}(\cdot)$ function, we have

$$
\operatorname{reg}(M)<\operatorname{Deg}(M)+\alpha(M)
$$

where $\alpha(M)$ is the maximal degree in a minimum graded generating set of $M$, and $\operatorname{reg}(M)$ is its Castelnuovo-Mumford regularity. 
Higher cohomology modules. Given a Gorenstein local ring $R$, and two finitely generated $R$-modules $A$ and $B$, we look at the problem of bounding the torsion of the modules $\operatorname{Tor}_{i}^{R}(A, B)$, for $i>0$. The approach we use is straightforward: Consider a free presentation,

$$
0 \rightarrow L \longrightarrow F \longrightarrow A \rightarrow 0
$$

pass to $L$ the given hdeg information on $A$, and use décalage to compare $h_{0}\left(\operatorname{Tor}_{i}^{R}(A, B)\right)$ to $h_{0}\left(\operatorname{Tor}_{i-1}^{R}(L, B)\right)$. This is allowed since by the cohomology exact sequences, we have the short exact sequences

$$
0 \rightarrow \operatorname{Tor}_{1}^{R}(A, B) \longrightarrow L \otimes B \longrightarrow F \otimes B \longrightarrow A \otimes B \rightarrow 0,
$$

and

$$
\operatorname{Tor}_{i}^{R}(A, B) \simeq \operatorname{Tor}_{i-1}^{R}(L, B), \quad i>1 .
$$

We relate the degrees of $A$ to those of $L$. We shall assume that the rank of $F$ is $\nu(A), \operatorname{dim} A=\operatorname{dim} R=d$, so that if $L \neq 0, \operatorname{dim} L=d$. This gives

$$
\operatorname{deg}(L)=\operatorname{deg}(F)-\operatorname{deg}(A) .
$$

We have the two expressions for $\operatorname{hdeg}(A)$ and $\operatorname{hdeg}(L)$ ([12, Definition 2.77]):

$$
\begin{aligned}
& \operatorname{hdeg}(A)=\operatorname{deg}(A)+\sum_{i=1}^{d}\left(\begin{array}{l}
d-1 \\
i-1
\end{array}\right) \cdot \operatorname{hdeg}\left(\operatorname{Ext}_{R}^{i}(A, R)\right), \\
& \operatorname{hdeg}(L)=\operatorname{deg}(L)+\sum_{i=1}^{d-1}\left(\begin{array}{l}
d-1 \\
i-1
\end{array}\right) \cdot \operatorname{hdeg}\left(\operatorname{Ext}_{R}^{i}(L, R)\right),
\end{aligned}
$$

since depth $L>0$. If we set $a_{i}=\operatorname{hdeg}\left(\operatorname{Ext}_{R}^{i}(A, R)\right)$, these formulas can be rewritten as

$$
\begin{aligned}
& \operatorname{hdeg}(A)=\operatorname{deg}(A)+a_{1}+\sum_{i=2}^{d}\left(\begin{array}{l}
d-1 \\
i-1
\end{array}\right) \cdot a_{i}, \\
& \operatorname{hdeg}(L)=\operatorname{deg}(L)+\sum_{i=1}^{d-1}\left(\begin{array}{c}
d-1 \\
i-1
\end{array}\right) \cdot a_{i+1}=\operatorname{deg}(L)+\sum_{i=1}^{d-1} \frac{i}{d-i}\left(\begin{array}{c}
d-1 \\
i
\end{array}\right) \cdot a_{i+1},
\end{aligned}
$$

where we have made use of the isomorphism $\operatorname{Ext}_{R}^{i}(A, R) \simeq \operatorname{Ext}_{R}^{i-1}(L, R)$, for $i>1$.

This gives

$$
\frac{1}{d-1} \sum_{i=2}^{d}\left(\begin{array}{c}
d-1 \\
i-1
\end{array}\right) \cdot a_{i} \leq \operatorname{hdeg}(L)-\operatorname{deg}(L) \leq(d-1) \cdot \sum_{i=2}^{d}\left(\begin{array}{c}
d-1 \\
i-1
\end{array}\right) \cdot a_{i} .
$$

We now collect these estimations: 
Proposition 2.6. Let $R, A$ and $L$ be as above. Then

$$
\operatorname{deg}(L) \leq \nu(A) \cdot \operatorname{deg}(R)-\operatorname{deg}(A),
$$

and if $c=\operatorname{hdeg}(A)-\operatorname{deg}(A)-\operatorname{hdeg}\left(\operatorname{Ext}_{R}^{1}(A, R)\right)$

$$
\frac{1}{d-1} c \leq \operatorname{hdeg}(L)-\operatorname{deg}(L) \leq(d-1) c .
$$

Theorem 2.7. Let $R$ be a Gorenstein local ring. If there is a polynomial $\mathbf{f}(x, y)$ such that for any two finitely generated $R$-modules $A, B$, in a certain class of modules, $h_{0}\left(A \otimes_{R} B\right) \leq \mathbf{f}(\operatorname{hdeg}(A), \operatorname{hdeg}(B))$, there are also polynomials $\mathbf{f}_{i}(x, y), i \geq 1$, of the same degree, such that

$$
h_{0}\left(\operatorname{Tor}_{i}^{R}(A, B)\right) \leq \mathbf{f}_{i}(\operatorname{hdeg}(A), \operatorname{hdeg}(B)) .
$$

Proof. Consider a minimal free presentation of $A$,

$$
0 \rightarrow L \longrightarrow F \longrightarrow A \rightarrow 0
$$

Tensoring with $B$, we have the exact sequence

$$
0 \rightarrow \operatorname{Tor}_{1}^{R}(A, B) \rightarrow L \otimes B \longrightarrow F \otimes B \longrightarrow A \otimes B \rightarrow 0
$$

hence $h_{0}\left(\operatorname{Tor}_{1}^{R}(A, B)\right) \leq h_{0}(L \otimes B)$. Now we make use of Proposition 2.6 to bound $h_{0}(L \otimes B)$ using the data on $A$.

The bounds for the higher Tor comes from the d'écalage.

\section{Dimension 1}

Suppose $R$ is a local domain of dimension 1 . We start our discussion with the case of two ideals, $I, J \subset R$. Consider the commutative diagram

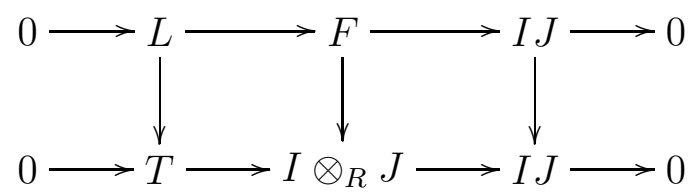

where $F$ is a free presentation of $I \otimes_{R} J$, and therefore it has rank $\nu(I) \cdot \nu(J)$. $L$ is a torsion free module with $\operatorname{rank}(L)=\operatorname{rank}(F)-1$, and therefore it can be generated by $\operatorname{deg}(L)=(\operatorname{rank}(F)-1) \operatorname{deg}(R)$ elements.

Proposition 3.1. If $R$ is a local domain of dimension one, essentially of finite type over a field and $I, J$ are $R$-ideals, then

$$
\begin{aligned}
h_{0}\left(I \otimes_{R} J\right) & \leq(\nu(I) \cdot \nu(J)-1) \operatorname{deg}(R) \cdot \lambda(R /(I, J, K)) \\
\nu\left(H_{\mathfrak{m}}^{0}\left(I \otimes_{R} J\right)\right) & \leq(\nu(I) \cdot \nu(J)-1) \operatorname{deg}(R),
\end{aligned}
$$

where $K$ is the Jacobian ideal of $R$.

We now quote in full two results [13] that we require for the proof. 
Theorem 3.2 ([13, Theorem 5.3]). Let $R$ be a Cohen-Macaulay local ring of dimension $d$, essentially of finite type over a field, and let $J$ be its Jacobian ideal. Then $J \cdot \operatorname{Ext}_{R}^{d+1}(M, \cdot)=0$ for any finitely generated $R$-module $M$, or equivalently, $J \cdot \operatorname{Ext}_{R}^{1}(M, \cdot)=0$ for any finitely generated maximal Cohen-Macaulay $R$-module $M$.

Proposition 3.3 ([13, Proposition 1.5]). Let $R$ be a commutative ring, $M$ and $R$ module, and $x \in R$. If $x \cdot \operatorname{Ext}_{R}^{1}(M, \cdot)=0$ then $x \cdot \operatorname{Tor}_{1}^{R}(M, \cdot)=0$.

Proof. The second formula arises because $L$ maps onto $T$, the torsion submodule of $I \otimes_{R} J$, which is also annihilated by $I, J$ and $K$. The last assertion is a consequence of the fact that $T=\operatorname{Tor}_{1}^{R}(I, R / J)$, and by Theorem 3.2 and Proposition $[3.3, K$ will annihilate it.

A small enhancement occurs since one can replace $I$ and $J$ by isomorphic ideals. In other words, the last factor, $\lambda(R /(I, J, K))$, can be replaced by $\lambda(R /(\tau(I), \tau(J), K))$, where $\tau(I)$ and $\tau(J)$ are their trace ideals $\left(\tau(I)=\right.$ image $I \otimes_{R} \operatorname{Hom}_{R}(I, R) \rightarrow R$ ).

The version for modules is similar:

Proposition 3.4. If $R$ is a local domain of dimension one, essentially of finite type over a field and let $A, B$ be finitely generated torsion free $R$-modules. Then

$$
\begin{aligned}
h_{0}\left(A \otimes_{R} B\right) & \leq(\nu(A) \cdot \nu(B)-\operatorname{rank}(A) \cdot \operatorname{rank}(B)) \operatorname{deg}(R) \cdot \lambda(R / K), \\
\nu\left(H_{\mathfrak{m}}^{0}\left(A \otimes_{R} B\right)\right) & \leq(\nu(A) \cdot \nu(B)-\operatorname{rank}(A) \cdot \operatorname{rank}(B)) \operatorname{deg}(R),
\end{aligned}
$$

where $K$ is the Jacobian ideal of $R$.

To extend this estimation of $h_{0}\left(A \otimes_{R} B\right)$ to finitely generated torsion free $R$-modules that takes into account annihilators we must equip the modules-as is the case of ideals-with a privileged embedding into free modules.

Proposition 3.5. Let $R$ be a Noetherian integral domain of dimension 1 , with finite integral closure. Let $A$ be a torsion free $R$-module of rank $r$ with the embedding $A \rightarrow F=R^{r}$. Let $I$ be the ideal image $\left(\wedge^{r} A \rightarrow \wedge F=R\right)$. Then $I$ annihilates $F / A$.

\section{VECTOR BUNDLES}

Let $(R, \mathfrak{m})$ be a regular local ring of dimension $d \geq 2$. For a vector bundle $A$ (that is, a finitely generated $R$-module that is free on the punctured spectrum), we establish estimates of the form

$$
h_{0}\left(A \otimes_{R} B\right) \leq c(R) \cdot \operatorname{hdeg}(A) \cdot \operatorname{hdeg}(B),
$$

where $c(R)$ is a constant depending on $R$.

From the general observations above, we may assume that $\operatorname{depth} A$ and $\operatorname{depth} B$ are positive. 
We make some reductions beginning with the following. Since $A$ is torsion free, consider the natural exact sequence

$$
0 \rightarrow A \longrightarrow A^{* *} \longrightarrow C \rightarrow 0 \text {. }
$$

Note that $C$ is a module of finite support, and that $A^{* *}$ is a vector bundle. Furthermore, since $A^{* *}$ has depth at least 2 , a direct calculation yields

$$
\operatorname{hdeg}(A)=\operatorname{hdeg}\left(A^{* *}\right)+\operatorname{hdeg}(C) .
$$

Tensoring the exact sequence by $B$, gives the exact complex

$$
\operatorname{Tor}_{1}^{R}(C, B) \longrightarrow A \otimes_{R} B \longrightarrow A^{* *} \otimes_{R} B,
$$

from which we obtain

$$
h_{0}\left(A \otimes_{R} B\right) \leq h_{0}\left(A^{* *} \otimes_{R} B\right)+\lambda\left(\operatorname{Tor}_{1}^{R}(C, B)\right) .
$$

As $C$ is a module of length $\operatorname{hdeg}(C)$,

$$
\lambda\left(\operatorname{Tor}_{1}^{R}(C, B)\right) \leq \beta_{1}(k) \cdot \operatorname{hdeg}(C) \cdot \nu(B) .
$$

We recall that $\nu(B) \leq \operatorname{hdeg}(B)$.

Let $(R, \mathfrak{m})$ be a Gorenstein local ring of dimension $d>0$ and let $A$ be a finitely generated $R$-module that is free on the punctured spectrum, and has finite projective dimension. We seek to estimate $h_{0}(A \otimes B)$ for various $R$-modules $B$.

Theorem 4.1. $B$ be a module of projective dimension $<d$ and let $A$ be a module that is free on the punctured spectrum. Then

$$
h_{0}(A \otimes B) \leq \sum_{i=0}^{d-1} \beta_{i}(B) \cdot h_{i}(A) .
$$

Proof. Let $B$ be an $R$-module with depth $B>0$ with the minimal free resolution

$$
0 \rightarrow F_{d-1} \longrightarrow F_{d-2} \longrightarrow \cdots \longrightarrow F_{1} \longrightarrow F_{0} \longrightarrow B \rightarrow 0 .
$$

Tensoring by $A$ gives a complex

$$
0 \rightarrow F_{d-1} \otimes A \longrightarrow F_{d-2} \otimes A \longrightarrow \cdots \longrightarrow F_{1} \otimes A \longrightarrow F_{0} \otimes A \longrightarrow B \otimes A \rightarrow 0,
$$

whose homology $H_{i}=\operatorname{Tor}_{i}(B, A), d>i>0$, has finite support. Denoting by $B_{i}$ and $Z_{i}$ its modules of boundaries and cycles, we have several exact sequences that start at $Z_{d-1}=0$

$$
\begin{aligned}
0 \rightarrow & B_{0} \longrightarrow F_{0} \otimes A \longrightarrow B \otimes A \rightarrow 0 \\
& 0 \rightarrow B_{i} \longrightarrow Z_{i} \longrightarrow H_{i} \rightarrow 0, \\
& \rightarrow Z_{i} \longrightarrow F_{i} \otimes A \longrightarrow B_{i-1} \rightarrow 0 .
\end{aligned}
$$

Taking local cohomology, we obtain the following acyclic complexes of modules of finite length

$$
H_{m}^{0}\left(F_{0} \otimes A\right) \longrightarrow H_{m}^{0}(B \otimes A) \longrightarrow H_{m}^{1}\left(B_{0}\right)
$$




$$
H_{m}^{i}\left(F_{i} \otimes A\right) \longrightarrow H_{m}^{i}\left(B_{i-1}\right) \longrightarrow H_{m}^{i+1}\left(Z_{i}\right), \quad d-1>i \geq 1 .
$$

Now we collect the inequalities of length, starting with

$$
h_{0}(B \otimes A) \leq \beta_{0}(B) \cdot h_{0}(A)+h_{1}\left(B_{0}\right)
$$

and

$$
h_{i}\left(B_{i-1}\right) \leq \beta_{i}(B) \cdot h_{i}(A)+h_{i+1}\left(Z_{i}\right) \leq \beta_{i}(B) \cdot h_{i}(A)+h_{i+1}\left(B_{i}\right),
$$

where we replace the rank of the modules $F_{i}$ by $\beta_{i}(B)$.

Theorem 4.2. Let $R$ be a regular local ring of dimension d. If $A$ is a finitely generated module free on the punctured spectrum, then for any finitely $R$-module $B$

$$
h_{0}(A \otimes B) \leq d \cdot \operatorname{hdeg}(A) \cdot \operatorname{hdeg}(B) .
$$

Proof. Let us rewrite the inequality in Theorem 4.1 in case $R$ is a regular local ring. Since by local duality $h_{i}(A)=\lambda\left(\operatorname{Ext}_{R}^{d-i}(A, R)\right)$ and $\beta_{i}(B) \leq \beta_{i}(k) \cdot \operatorname{hdeg}(B)$, we have

$$
\begin{aligned}
h_{0}(A \otimes B) & \leq \operatorname{hdeg}(B) \cdot \sum_{i=0}^{d-1}\left(\begin{array}{c}
d \\
i
\end{array}\right) \lambda\left(\operatorname{Ext}_{R}^{d-i}(A, R)\right) \\
& \leq d \cdot \operatorname{hdeg}(B) \sum_{i=1}^{d}\left(\begin{array}{c}
d-1 \\
i-1
\end{array}\right) \lambda\left(\operatorname{Ext}_{R}^{d-i}(A, R)\right) \\
& \leq d \cdot \operatorname{hdeg}(A) \cdot \operatorname{hdeg}(B) .
\end{aligned}
$$

Finally, to deal with a general module $B$, it suffices to add to hdeg $(B)$ the correction $h_{0}(B)$ as given in Proposition 2.1.

Example 4.3. Let $(R, \mathfrak{m})$ be a Gorenstein local ring of dimension $d \geq 1$ and let $A$ be a module with a presentation

$$
0 \rightarrow R^{n} \stackrel{\varphi}{\longrightarrow} R^{n+d-1} \longrightarrow A \rightarrow 0,
$$

where the ideal $I_{n}(\varphi)$ is m-primary. $A$ is a vector bundle of projective dimension 1 . According to a well-known length formula $([3]), \lambda\left(\operatorname{Ext}_{R}^{1}(A, R)\right)=\lambda\left(R / I_{n}(\varphi)\right)$, from which it follows that

$$
\begin{aligned}
\operatorname{hdeg}(A) & =(d-1) \operatorname{deg}(A)+\lambda\left(R / I_{n}(\varphi)\right), \quad \text { and therefore } \\
h_{0}(A \otimes A) & \leq d\left((d-1) \operatorname{deg}(A)+\lambda\left(R / I_{n}(\varphi)\right)\right)^{2} .
\end{aligned}
$$

\section{Dimension 2}

Let $(R, \mathfrak{m})$ be a regular local ring of dimension 2 (or a polynomial ring $k[x, y]$ over the field $k)$. For two $R$-modules $A$ and $B$ we are going to study $h_{0}\left(A \otimes_{R} B\right)=$ $\lambda\left(H_{\mathfrak{m}}^{0}\left(A \otimes_{R} B\right)\right)$ through a series of reductions on $A, B$ and $R$. Now we examine how the presence of torsion affects the analysis.

Eventually the problem will settle on the consideration of a special class of onedimensional rings. 
We are already familiar with the stripping away from $A$ and $B$ of their submodules of finite support, so we may assume that these modules have $\operatorname{depth} \geq 1$. Let $A$ be a module of dimension 2 , and denote by $A_{0}$ the torsion submodule of $A$. Consider the natural exact sequence

$$
0 \rightarrow A_{0} \longrightarrow A \longrightarrow A^{\prime} \rightarrow 0
$$

with $A^{\prime}$ torsion free. If $A_{0} \neq 0$, it is a Cohen-Macaulay module of dimension 1 . We have the exact sequence

$$
0 \rightarrow \operatorname{Ext}_{R}^{1}\left(A^{\prime}, R\right) \longrightarrow \operatorname{Ext}_{R}^{1}(A, R) \longrightarrow \operatorname{Ext}_{R}^{1}\left(A_{0}, R\right) \rightarrow 0,
$$

that yields

$$
\begin{aligned}
\operatorname{deg} A & =\operatorname{deg} A^{\prime} \\
\operatorname{hdeg}\left(\operatorname{Ext}_{R}^{1}(A, R)\right) & =\operatorname{hdeg}\left(\operatorname{Ext}_{R}^{1}\left(A^{\prime}, R\right)\right)+\operatorname{deg} A_{0},
\end{aligned}
$$

in particular

$$
\operatorname{hdeg}(A)=\operatorname{deg} A_{0}+\operatorname{hdeg}\left(A^{\prime}\right)
$$

As a consequence, $\operatorname{hdeg}\left(A^{\prime}\right)$ and $\operatorname{hdeg}\left(A_{0}\right)$ are bounded in terms of hdeg $(A)$. Now we tensor the sequence by $B$ to get the complex

$$
\operatorname{Tor}_{1}^{R}\left(A^{\prime}, B\right) \longrightarrow A_{0} \otimes_{R} B \longrightarrow A \otimes_{R} B \longrightarrow A \otimes_{R} B \rightarrow 0 .
$$

If we denote by $L$ the image of $A_{0} \otimes_{R} B$ in $A \otimes_{R} B$, since $\operatorname{Tor}_{1}^{R}\left(A^{\prime}, B\right)$ is a module of finite length, we have

$$
\begin{aligned}
h_{0}\left(A \otimes_{R} B\right) & \leq h_{0}\left(A^{\prime} \otimes_{R} B\right)+h_{0}(L), \\
h_{0}(L) & \leq h_{0}\left(A_{0} \otimes_{R} B\right) .
\end{aligned}
$$

If we apply a similar reduction to $B$ and combine, we get

$$
h_{0}\left(A \otimes_{R} B\right) \leq h_{0}\left(A^{\prime} \otimes_{R} B^{\prime}\right)+h_{0}\left(A_{0} \otimes_{R} B^{\prime}\right)+h_{0}\left(A^{\prime} \otimes_{R} B_{0}\right)+h_{0}\left(A_{0} \otimes_{R} B_{0}\right) .
$$

A term like $h_{0}\left(A^{\prime} \otimes B_{0}\right)$ is easy to estimate since $A^{\prime}$ is a vector bundle of low dimension. For convenience, let

$$
0 \rightarrow F_{1} \longrightarrow F_{0} \longrightarrow A^{\prime} \rightarrow 0
$$

be a minimal resolution of $A^{\prime}$. Tensoring with $B_{0}$, we get the exact sequence

$$
0 \rightarrow \operatorname{Tor}_{1}^{R}\left(A^{\prime}, B_{0}\right) \longrightarrow F_{1} \otimes_{R} B_{0} \longrightarrow F_{0} \otimes_{R} B_{0} \longrightarrow A^{\prime} \otimes_{R} B_{0} \rightarrow 0 .
$$

Since $\operatorname{Tor}_{1}^{R}\left(A^{\prime}, B_{0}\right)$ has finite support and $F_{1} \otimes_{R} B_{0}$ has positive depth, $\operatorname{Tor}_{1}^{R}\left(A^{\prime}, B_{0}\right)=$ 0 . To compute $h_{0}\left(A^{\prime} \otimes_{R} B_{0}\right)$, consider a minimal resolution of $B_{0}$,

$$
0 \rightarrow G \longrightarrow G \longrightarrow B_{0} \rightarrow 0,
$$

and the exact sequence

$$
0 \rightarrow A^{\prime} \otimes_{R} G \longrightarrow A^{\prime} \otimes_{R} G \longrightarrow A^{\prime} \otimes_{R} B_{0} \rightarrow 0 .
$$


From the cohomology exact sequence, we have the surjection

$$
\operatorname{Ext}_{R}^{1}\left(A^{\prime} \otimes G, R\right) \longrightarrow \operatorname{Ext}_{R}^{2}\left(A^{\prime} \otimes_{R} B_{0}\right),
$$

and therefore, since $\operatorname{Ext}_{R}^{1}(A, R)$ is a module of finite support,

$$
h_{0}\left(A^{\prime} \otimes_{R} B_{0}\right) \leq \nu(G) \cdot \lambda\left(\operatorname{Ext}_{R}^{1}\left(A^{\prime}, R\right)\right) .
$$

This shows that

$$
h_{0}\left(A^{\prime} \otimes_{R} B_{0}\right) \leq \nu(G) \cdot\left(\operatorname{hdeg}\left(A^{\prime}\right)-\operatorname{deg} A^{\prime}\right)<\operatorname{hdeg}\left(A^{\prime}\right) \cdot \operatorname{hdeg}\left(B_{0}\right) .
$$

The reductions thus far lead us to assume that $A$ and $B$ are $R$-modules of positive depth and dimension 1 . Let

$$
0 \rightarrow F \stackrel{\varphi}{\longrightarrow} F \longrightarrow A \rightarrow 0
$$

be a minimal free resolution of $A$. By a standard calculation,

$$
\operatorname{deg} A=\operatorname{deg}(R / \operatorname{det}(\varphi)) .
$$

Since $\operatorname{det}(\varphi)$ annihilates $A$, we could view $A \otimes_{R} B$ as a module of over $R /(\operatorname{det}(\varphi \circ \psi))$ where $\psi$ is the corresponding matrix in the presentation of $B$.

To avoid dealing with two matrices, replacing $A$ by $A \oplus B$, we may consider $h_{0}\left(A \otimes_{R}\right.$ $A$ ), but still denote by $\varphi$ the presentation matrix (instead of $\varphi \oplus \psi$ ), and set $S=$ $R /(\operatorname{det}(\varphi))$; note that $\operatorname{deg} S=\operatorname{deg} A$.

Example 5.1. We consider a cautionary family of examples to show that other numerical readings must be incorporated into the estimates for $h_{0}\left(A \otimes_{R} A\right)$.

Let $A$ be a module generated by two elements, with a free resolution

$$
0 \rightarrow F \stackrel{\varphi}{\longrightarrow} F \longrightarrow A \rightarrow 0 \text {. }
$$

Suppose $k$ is a field of characteristic $\neq 2$. To calculate $h_{0}\left(A \otimes_{R} A\right)$, we make use of the decomposition

$$
A \otimes_{R} A=S_{2}(A) \oplus \wedge^{2} A .
$$

Given a matrix representation,

$$
\varphi=\left[\begin{array}{ll}
a_{11} & a_{12} \\
a_{21} & a_{22}
\end{array}\right]
$$

one has

$$
\wedge^{2} A \simeq R / I_{1}(\varphi)=R /\left(a_{11}, a_{12}, a_{21}, a_{22}\right) .
$$

The symmetric square of $A, S_{2}(A)$, has a resolution

$$
0 \rightarrow R \stackrel{\phi}{\longrightarrow} F \otimes_{R} F \stackrel{\psi}{\longrightarrow} S_{2}(F),
$$

where

$$
\begin{aligned}
\psi(u \otimes v) & =u \cdot \varphi(v)+v \cdot \varphi(u) \\
\phi(u \wedge v) & =\varphi^{\prime}(u) \otimes v-\varphi^{\prime}(v) \otimes u
\end{aligned}
$$


where $\varphi^{\prime}$ is the matrix obtained from $\varphi$ by dividing out its entries by their gcd $a$, $\varphi=a \cdot \varphi^{\prime}$.

A straightforward calculation will give

$$
\operatorname{Ext}_{R}^{2}\left(S_{2}(A), R\right)=R / I_{1}\left(\varphi^{\prime}\right)
$$

This shows that

$$
\begin{aligned}
h_{0}\left(A \otimes_{R} A\right) & =h_{0}\left(R / I_{1}(\varphi)\right)+h_{0}\left(R / I_{1}\left(\varphi^{\prime}\right)\right) \\
& =h_{0}\left(a R / a I_{1}\left(\varphi^{\prime}\right)\right)+h_{0}\left(R / I_{1}\left(\varphi^{\prime}\right)\right)=2 \cdot \lambda\left(R / I_{1}\left(\varphi^{\prime}\right)\right) .
\end{aligned}
$$

Thus the matrix

$$
\varphi=\left[\begin{array}{ll}
x & y^{n} \\
0 & x
\end{array}\right]
$$

will define a module $A$, with $\operatorname{deg}(A)=2$, but $h_{0}\left(A \otimes_{R} A\right)=2 n$. This means that we must take into account the degrees of the entries of $\varphi$ itself.

Example 5.2. Let $R$ be a Cohen-Macaulay local ring and let $\left\{x_{1}, \ldots, x_{n}\right\}, n \geq 2$, be a set of elements such that any pair forms a regular sequence. Set

$$
\begin{aligned}
\mathbf{x} & =x_{1} \cdots x_{n}, \\
z_{i} & =\mathbf{x} / x_{i}, \quad i=1 \ldots n .
\end{aligned}
$$

We claim that

$$
\operatorname{deg}\left(R /\left(z_{1}, \ldots, z_{n}\right)\right) \leq \frac{1}{2}\left((\operatorname{deg}(R /(\mathbf{x})))^{2}-n\right) .
$$

We argue by induction on $n$, the formula being clear for $n=2$.

Consider the exact sequence

$$
0 \rightarrow\left(x_{1}, z_{2}, \ldots, z_{n}\right) /\left(z_{1}, z_{2}, \ldots, z_{n}\right) \longrightarrow R /\left(z_{1}, z_{1}\right) \longrightarrow R /\left(x_{1}, z_{1}\right) \rightarrow 0 .
$$

Since

$$
\left(x_{1}, z_{1}\right) /\left(z_{1}, \ldots, z_{n}\right) \simeq R /\left(z_{2}^{\prime}, \ldots, z_{n}^{\prime}\right),
$$

where $z_{i}^{\prime}, i \geq 2$, denotes the products from elements in the set $\left\{x_{2}, \ldots, x_{n}\right\}$ using the formation rule of the $z_{i}$.

Adding the multiplicities of the modules of the same dimension, we have

$$
\operatorname{deg}\left(R /\left(z_{1}, \ldots, z_{n}\right)\right)=\operatorname{deg}\left(R /\left(z_{1}, \ldots, z_{n}\right)\right)+\operatorname{deg}\left(R /\left(z_{2}^{\prime}, \ldots, z_{n}^{\prime}\right)\right) .
$$

As

$$
\operatorname{deg}\left(R /\left(x_{1}, z_{1}\right)\right)=\operatorname{deg}\left(R /\left(x_{1}\right)\right) \cdot \operatorname{deg}\left(R /\left(z_{1}\right)\right)=\operatorname{deg}\left(R /\left(x_{1}\right)\right) \cdot \sum_{j \geq 2} \operatorname{deg}\left(R /\left(x_{j}\right)\right),
$$

and by induction

$$
\operatorname{deg}\left(R /\left(z_{2}^{\prime}, \ldots, z_{n}^{\prime}\right)\right)=\sum_{2 \leq i<j \leq n} \operatorname{deg}\left(R /\left(x_{i}\right)\right) \cdot \operatorname{deg}\left(R /\left(x_{j}\right)\right),
$$


we have

$$
\operatorname{deg}\left(R /\left(z_{1}, \ldots, z_{n}\right)=\sum_{1 \leq i<j \leq n} \operatorname{deg}\left(R /\left(x_{i}\right)\right) \cdot \operatorname{deg}\left(R /\left(x_{j}\right)\right) .\right.
$$

The rest of the calculation is clear. There are similar formulas in case every subset of $k$ elements of $\left\{x_{1}, \ldots, x_{n}\right\}$ forms a regular sequence.

Now we return to the modules with a presentation

$$
0 \rightarrow F \stackrel{\varphi}{\longrightarrow} F \longrightarrow A \rightarrow 0
$$

and write $\operatorname{det}(\varphi)=\mathbf{x}=x_{1} \cdots x_{n}$. Setting $z_{i}=\mathbf{x} / x_{i}$, consider the exact sequence

$$
0 \rightarrow R /(\mathbf{x}) \longrightarrow R /\left(z_{1}\right) \oplus \cdots \oplus R /\left(z_{n}\right) \longrightarrow C \rightarrow 0
$$

induced by the mapping $1 \mapsto\left(z_{1}, \ldots, z_{n}\right)$. $C$ is a module of finite length, and making use of duality and the inequality (7),

$$
\lambda(C) \leq \frac{1}{2}\left(\left(\operatorname{deg}(R /(\mathbf{x}))^{2}-n\right) .\right.
$$

Tensoring this sequence by $A$, gives

$$
\operatorname{Tor}_{1}^{R}(A, C) \longrightarrow A \longrightarrow A_{1} \oplus \cdots \oplus A_{n} \longrightarrow A \otimes_{R} C \rightarrow 0
$$

and since depth $A>0$, we have the exact sequence

$$
0 \rightarrow A \longrightarrow A_{1} \oplus \cdots \oplus A_{n} \longrightarrow A \otimes_{R} C \rightarrow 0,
$$

where $A_{i}=A / x_{i} A$ and $\lambda(A \otimes C) \leq \nu(A) \lambda(C)$. These relations give that

$$
\begin{aligned}
\operatorname{deg} A & =\sum_{i=1}^{n} \operatorname{deg} A_{i} \\
\lambda\left(A \otimes_{R} C\right) & \geq \sum_{i=1}^{n} h_{0}\left(A_{i}\right) .
\end{aligned}
$$

These inequalities show that we are still tracking the $\operatorname{hdeg}\left(A_{i}\right)$ in terms of $\operatorname{deg} A$.

Tensoring the last exact sequence by $A$, we obtain the exact complex

$$
\operatorname{Tor}_{1}^{R}\left(A, A \otimes_{R} C\right) \longrightarrow A \otimes_{R} A \longrightarrow A_{1} \otimes_{R} A_{1} \oplus \cdots \oplus A_{n} \otimes_{R} A_{n},
$$

from which we have

$$
\begin{aligned}
h_{0}\left(A \otimes_{R} A\right) & \leq \sum_{i=1}^{n} h_{0}\left(A_{i} \otimes_{R} A_{i}\right)+\lambda\left(\operatorname{Tor}_{1}^{R}\left(A, A \otimes_{R} C\right)\right) \\
& \leq \sum_{i=1}^{n} h_{0}\left(A_{i} \otimes_{R} A_{i}\right)+\beta_{1}(A) \cdot \nu(A) \cdot \lambda(C) .
\end{aligned}
$$

Let us sum up these reductions as follows: 
Proposition 5.3. Let $R$ be a two-dimensional regular local ring and let $A$ be a CohenMacaulay R-module of dimension one. Then

$$
h_{0}\left(A \otimes_{R} A\right) \leq 3 \cdot \operatorname{hdeg}(A)^{4},
$$

provided

$$
h_{0}\left(A \otimes_{R} A\right) \leq 2 \cdot \operatorname{hdeg}(A)^{4}
$$

whenever $\operatorname{ann} A$ is a primary ideal.

Proof. Note that $\beta_{1}(A) \leq \beta_{1}(k) \cdot \operatorname{hdeg}(A), \nu(A) \leq \operatorname{hdeg}(A)$, and $\lambda(C)<\frac{1}{2} \operatorname{hdeg}(A)^{2}$.

\section{Dimension 3}

The technique of Theorem 4.2 can be used to deal with torsionfree modules of dimension three.

Theorem 6.1. Let $R$ be a regular local ring of dimension 3, and let $A$ and $B$ be torsionfree $R$-modules. Then

$$
h_{0}(A \otimes B)<4 \cdot \operatorname{hdeg}(A) \cdot \operatorname{hdeg}(B) .
$$

Proof. Consider the natural exact sequence

$$
0 \rightarrow A \longrightarrow A^{* *} \longrightarrow C \rightarrow 0 .
$$

A straightforward calculation will show that

$$
\operatorname{hdeg}(A)=\operatorname{hdeg}\left(A^{* *}\right)+\operatorname{hdeg}(C) .
$$

Note that $A^{* *}$ is a vector bundle of projective dimension at most 1 by the AuslanderBuchsbaum equality ([2, Theorem 1.3.3]), and $C$ is a module of dimension at most 1 . Tensoring by the torsionfree $R$-module $B$, we have the exact sequence

$$
\operatorname{Tor}_{1}^{R}\left(A^{* *}, B\right) \longrightarrow \operatorname{Tor}_{1}(C, B) \longrightarrow A \otimes B \longrightarrow A^{* *} \otimes B \longrightarrow C \otimes B \rightarrow 0,
$$

where $\operatorname{Tor}_{1}^{R}\left(A^{* *}, B\right)=0$, since proj $\operatorname{dim} A^{* *} \leq 1$ and $B$ is torsionfree.

From the exact sequence, we have

$$
h_{0}(A \otimes B) \leq h_{0}\left(A^{* *} \otimes B\right)+h_{0}\left(\operatorname{Tor}_{1}^{R}(C, B)\right) .
$$

Because $A^{* *}$ is a vector bundle, by Theorem 4.2 ,

$$
h_{0}\left(A^{* *} \otimes B\right) \leq 3 \cdot \operatorname{hdeg}\left(A^{* *}\right) \cdot \operatorname{hdeg}(B) .
$$

For the module $\operatorname{Tor}_{1}^{R}(C, B)$, from a minimal free presentation of $B$,

$$
0 \rightarrow L \longrightarrow F \longrightarrow B \rightarrow 0
$$

we have an embedding $\operatorname{Tor}_{1}^{R}(C, B) \rightarrow C \otimes L$, and therefore

$$
h_{0}\left(\operatorname{Tor}_{1}^{R}(C, B)\right) \leq h_{0}(C \otimes L) \leq 3 \cdot \operatorname{hdeg}(L) \cdot \operatorname{hdeg}(C),
$$


because $L$ is a vector bundle. In turn

$$
\begin{aligned}
\operatorname{deg}(L) & =\beta_{1}(B)-\operatorname{deg}(B) \leq \beta_{1}(R / \mathfrak{m}) \cdot \operatorname{hdeg}(B)-\operatorname{deg}(B) \\
& =3 \cdot \operatorname{hdeg}(B)-\operatorname{deg}(B)
\end{aligned}
$$

by Theorem 2.4, and since $\operatorname{Ext}_{R}^{1}(L, R)=\operatorname{Ext}_{R}^{2}(B, R)$,

$$
\operatorname{hdeg}(L)=\operatorname{deg}(L)+\operatorname{hdeg}\left(\operatorname{Ext}_{R}^{1}(L, R)\right)<4 \cdot \operatorname{hdeg}(B) .
$$

Finally we collect (11) and (12) into (10), along with (9),

$$
\begin{aligned}
h_{0}(A \otimes B) & <3 \cdot \operatorname{hdeg}\left(A^{* *}\right) \cdot \operatorname{hdeg}(B)+4 \cdot \operatorname{hdeg}(B) \cdot \operatorname{hdeg}(C) \\
& <4 \cdot \operatorname{hdeg}(A) \cdot \operatorname{hdeg}(B),
\end{aligned}
$$

as asserted.

\section{Graded MOdules}

We give a rough (high degree) estimate for the case of graded modules over $R=$ $k[x, y]$. We may assume that $A$ is not a cyclic module. Furthermore, we shall assume that $A$ is equi-generated.

We briefly describe the behavior of $\operatorname{reg}(\cdot)$ with regard to some exact sequences.

Proposition 7.1. Let $R$ be a standard graded algebra, and let

$$
0 \rightarrow A \longrightarrow B \longrightarrow C \rightarrow 0
$$

be an exact sequence of finitely generated graded $R$-modules, then

$$
\operatorname{reg}(B) \leq \operatorname{reg}(A)+\operatorname{reg}(C)
$$

Similarly,

$$
\operatorname{reg}(A) \leq \operatorname{reg}(B)+\operatorname{reg}(C), \quad \text { and } \quad \operatorname{reg}(C) \leq \operatorname{reg}(A)+\operatorname{reg}(B) .
$$

Let us apply it to the graded $k[x, y]$-module $A$ of depth $>0$. In the exact sequence

$$
0 \rightarrow A_{0} \longrightarrow A \longrightarrow A^{\prime} \rightarrow 0
$$

we already remarked that $\operatorname{hdeg}(A)=\operatorname{hdeg}\left(A^{\prime}\right)+\operatorname{deg} A_{0}$. It is also the case that if $A$ is generated by elements of degree $\leq \alpha(A)$, then by the proposition above and Theorem 2.5, $\operatorname{reg}\left(A_{0}\right)<\operatorname{hdeg}(A)+\alpha(A)$. Actually, since $A_{0}$ is Cohen-Macaulay, a direct calculation will show that $\operatorname{reg}\left(A_{0}\right) \leq \operatorname{reg}(A)$.

We may assume that $A$ is a one-dimensional graded $R$-module with a minimal resolution

$$
0 \rightarrow F \stackrel{\varphi}{\longrightarrow} F \longrightarrow A \rightarrow 0 .
$$

A presentation of $A \otimes_{R} A$ is given by

$$
F \otimes_{R} F \oplus F \otimes_{R} F \stackrel{\psi}{\longrightarrow} F \otimes_{R} F,
$$


where $\psi=\varphi \otimes I-I \otimes \varphi$. The kernel of $\psi$ contains the image of

$$
\phi: F \otimes_{R} F \longrightarrow F \otimes_{R} F \oplus F \otimes_{R} F, \quad \phi=I \otimes \varphi \oplus \varphi \otimes I .
$$

Since $R=k[x, y], L=\operatorname{ker}(\psi)$ is a free $R$-module of rank $r^{2}, r=\operatorname{rank}(F)$. Because $\phi$ is injective, its image $L_{0}$ is a free $R$-submodule of $\mathbf{F}=F \otimes_{R} F \oplus F \otimes_{R} F$ of the same rank as $L, L_{0} \subset L, \phi^{\prime}: F \otimes F \rightarrow \mathbf{F}$. It follows that the degrees of the entries of $\phi^{\prime}$ cannot be higher than those of $\phi$.

To estimate $h_{0}\left(A \otimes_{R} A\right)$, note that $\operatorname{Ext}_{R}^{2}\left(A \otimes_{R} A, R\right)$ is the cokernel of map $\phi$. This is a module generated by $r^{2}$ elements, annihilated by the maximal minors of $\phi$. We already have that $\mathbf{f}=\operatorname{det}(\varphi)$ annihilates $A$. Now we look for an element $\mathbf{h}$ in the ideal of maximal minors of $\phi^{\prime}$ so that $(\mathbf{f}, \mathbf{h})$ has finite colength, and as a consequence we would have

$$
h_{0}\left(A \otimes_{R} A\right) \leq r^{2} \cdot \lambda(R /(\mathbf{f}, \mathbf{h}))=r^{2} \cdot \operatorname{deg}(R /(\mathbf{f})) \operatorname{deg}(R /(\mathbf{h})) .
$$

The entries of $\varphi$ have degree $\leq \operatorname{deg}(A)-1$, so the minors $\mathbf{h}$ of $\phi$ have degree

$$
\operatorname{deg} \mathbf{h} \leq r^{2} \cdot(\operatorname{deg}(A)-1)
$$

Proposition 7.2. If $A$ is a graded $k[x, y]$-module of dimension 1 , equigenerated in degree 0 , then

$$
h_{0}\left(A \otimes_{R} A\right) \leq r^{4} \cdot \operatorname{deg}(A)(\operatorname{deg}(A)-1)<\operatorname{deg}(A)^{6}
$$

\section{Some OPEn QUESTIONS}

There are numerous open issues regarding the torsion in tensor products that are not discussed here. We raise a few related to the discussion above.

(1) How good are some of the estimates for $h_{0}(A \otimes A)$ compared to actual values, for instance of Example 4.3?

(2) In [5], Theorem 3.2 is used to extend the HomAB version of Theorem 4.2 from the regular to the isolated singularity case. Is there a similar extension to $h_{0}(A \otimes B)$ ?

(3) How to derive more general estimates in dimension 3, particularly of graded modules with torsion?

(4) Let $R$ be a Noetherian local domain and $A$ a finitely generated torsionfree $R$-module. Is there an integer $e=e(R)$ guaranteeing that if $M$ is not $R$-free, then the tensor power $M^{\otimes e}$ has nontrivial torsion? The motivation is a result of Auslander ([1], see also [8]) that asserts that $e=\operatorname{dim} R$ works for all regular local rings. For instance, if $R$ is a one-dimensional domain, will $e=2$ work? A more realistic question is, if $R$ is a Cohen-Macaulay local domain of dimension $d$ and multiplicity $\mu$, will

$$
e=d+\mu-1
$$


suffice? Note that if we make no attempt to determine uniform bounds for $e$, if $\mathbb{Q} \subset R$, then for a module $M$ of rank $r$ and minimal number of generators $n$, then the embedding

$$
0 \neq \wedge^{n} M \hookrightarrow M^{\otimes n}
$$

shows the existence of a test power for $M$.

\section{REFERENCES}

[1] Auslander, M. Modules over unramified regular local rings. Illinois J. Math. 5 (1961), 631-647.

[2] Bruns, W, Herzog, J. Cohen-Macaulay Rings, Cambridge University Press, 1993.

[3] Bruns, W., Vetter, U. Length formulas for the local cohomology of exterior powers. Math. Zeitshcrift 191 (1986), 145-158.

[4] Dalili, K. On the number of generators of modules of homomorphisms. J. Algebra 311 (2007), 463-491.

[5] Dalili, K., Vasconcelos, W.V. Cohomological degrees and the HomAB conjecture. In Algebra, Geometry and Their Interactions (A. Corso, J. Migliore and C. Polini Eds.), Contemp. Math. 448 (2007), 43-61.

[6] Doering, L.R., Gunston, T., Vasconcelos, W. V. Cohomological degrees and Hilbert functions of graded modules. American J. Math. 120 (1998), 493-504.

[7] Huneke, C., Wiegand, R. Tensor products of modules and the rigidity of Tor. Math. Annalen 299 (1994), 449-476. Correction, Math. Annalen 338 (2007), 291-293.

[8] Lichtenbaum, S. On the vanishing of Tor in regular local rings. Illinois J. Math. 10 (1966), $220-226$.

[9] Nagel, U. Comparing Castelnuovo-Mumford regularity and extended degree: the borderline cases. Trans. Amer. Math. Soc. 357 (2005), 3585-3603.

[10] Vasconcelos, W. V. Arithmetic of Blowup Algebras. London Math. Soc., Lecture Note Series 195, Cambridge University Press, 1994.

[11] Vasconcelos, W. V. The homological degree of a module. Trans. Amer. Math. Soc. 350 (1998), $1167-1179$.

[12] Vasconcelos, W. V. Integral Closure. Springer Monographs in Mathematics, New York, 2005.

[13] Wang, H.-J. On the Fitting ideals of free resolutions. Michigan Math. J. 41 (1994), 587-608.

Department of Mathematics, Rutgers University

110 Frelinghuysen Rd, Piscataway, NJ 08854-8019, U.S.A.

E-mail address: vasconce@math.rutgers.edu 